\title{
Generation of picosecond pulses and frequency combs in actively mode locked external ring cavity quantum cascade lasers
}

\section{Citation}

Wójcik, Aleksander K., Pietro Malara, Romain Blanchard, Tobias S. Mansuripur, Federico Capasso, and Alexey Belyanin. 2013. "Generation of Picosecond Pulses and Frequency Combs in Actively Mode Locked External Ring Cavity Quantum Cascade Lasers." Applied Physics Letters 103 (23): 231102. https://doi.org/10.1063/1.4838275.

\section{Permanent link}

http://nrs.harvard.edu/urn-3:HUL.InstRepos:41371602

\section{Terms of Use}

This article was downloaded from Harvard University's DASH repository, and is made available under the terms and conditions applicable to Other Posted Material, as set forth at http:// nrs.harvard.edu/urn-3:HUL.InstRepos:dash.current.terms-of-use\#LAA

\section{Share Your Story}

The Harvard community has made this article openly available.

Please share how this access benefits you. Submit a story.

\section{Accessibility}




\title{
Generation of picosecond pulses and frequency combs in actively mode locked external ring cavity quantum cascade lasers
}

\author{
Aleksander K. Wójcik, ${ }^{1}$ Pietro Malara, ${ }^{2,3}$ Romain Blanchard, ${ }^{2}$ Tobias S. Mansuripur, ${ }^{2}$ \\ Federico Capasso, ${ }^{2}$ and Alexey Belyanin ${ }^{1, a)}$ \\ ${ }^{1}$ Department of Physics and Astronomy, Texas A\&M University, College Station, Texas 77843-4242, USA \\ ${ }^{2}$ School of Engineering and Applied Sciences, Harvard University, Cambridge, Massachusetts 02138, USA \\ ${ }^{3}$ Consiglio Nazionale delle Ricerche-Istituto Nazionale di Ottica, and European Laboratory for Nonlinear \\ Spectroscopy (LENS), 80078 Pozzuoli (NA), Italy
}

(Received 2 September 2013; accepted 17 November 2013; published online 2 December 2013)

\begin{abstract}
We propose a robust and reliable method of active mode locking of mid-infrared quantum cascade lasers and develop its theoretical description. Its key element is the use of an external ring cavity, which circumvents fundamental issues undermining the stability of mode locking in quantum cascade lasers. We show that active mode locking can give rise to the generation of picosecond pulses and phase-locked frequency combs containing thousands of the ring cavity modes. (C) 2013 AIP Publishing LLC. [http://dx.doi.org/10.1063/1.4838275]
\end{abstract}

Generation of ultrashort pulses in mid-infrared quantum cascade lasers (QCLs) has proven to be challenging. The fast gain recovery essentially prohibits passive mode locking with a saturable absorber. Ultrashort and high-power pulses cannot be obtained by gain switching in the absence of relaxation oscillations; the observed duration of gain-switched pulses was limited to hundreds of picoseconds. ${ }^{1}$ Although picosecond range pulses have been obtained in an actively modulated two-section QCL close to laser threshold, ${ }^{2}$ active mode locking in monolithic Fabry-Perot lasers appears to be limited by inherent multimode instabilities of the QCLs. The multimode behavior originates from the spatial hole burning (SHB) inside the cavity, which leads to the formation of a transient population grating on the intersubband gain transition. This grating dissipates by carrier diffusion at a slower rate than the gain recovers, in contrast to bipolar semiconductor diode lasers. Scattering of the laser modes off the population grating leads to the proliferation of modes with uncorrelated phases, which destroys single-pulse operation and leads to a chaotic output. ${ }^{2-4}$ The situation is more favorable in terahertz $(\mathrm{THz})$ QCLs due to a longer gain recovery time. Active mode locking in THz QCLs was reported, e.g., in Refs. 5 and 6.

While the conditions for ultrashort pulse generation in mid-infrared QCLs are highly unfavorable, stable phase locking between laser modes is feasible. For example, Ref. 7 observed spectra with a narrow intermode beat and reported the evidence of stable phase locking between longitudinal modes. Phase-locked frequency combs in QCLs supporting several lateral modes were reported and theoretically modeled in Refs. 8-10. Theoretical studies ${ }^{9,10}$ implicate weak waveguide dispersion, fast gain relaxation, and strong thirdorder resonant nonlinearity associated with the gain transition as the main reasons for stable phase locking. However, the resulting phase relationships between laser modes correspond to a frequency modulated signal with a quasi-constant intensity. ${ }^{7}$ As expected, no stable pulses were observed in any of these studies.

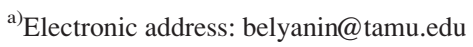

The use of a unidirectional external ring cavity prevents the onset of the SHB multimode instability and facilitates active mode locking with stable ultrashort pulse operation. Furthermore, in external-cavity lasers, one can achieve a deep modulation of the gain and at the same time creates a very short gain window as compared to the round-trip time. In monolithic QCLs, one has to apply gain modulation to a short section of the laser. ${ }^{2}$ The resulting modulation depth will be low and will lead to mode locking only near threshold. In addition, a long external cavity has round-trip frequencies reduced from tens of gigahertz in monolithic QCLs to hundreds of megahertz, which is much more convenient for gain or loss modulation and makes it easy to incorporate additional optical elements or a gas cell inside the cavity.

We study theoretically the formation of picosecond midinfrared pulses by direct modulation of the gain in an external ring-cavity QCL (ERC-QCL). We show that active mode locking in ERC-QCLs results in robust, stable picosecond pulses, and phase-locked frequency combs at injection currents considerably above threshold. We establish the limits to the pulse duration and power for typical parameters of mid-infrared QCLs. Although there is a tradeoff between the pulse duration and power, the shortest pulses can be further amplified outside the laser cavity or used in spectroscopy applications that do not require high power.

The unidirectional lasing on ring-cavity modes in QCLs has been recently demonstrated in both a monolithically integrated design ${ }^{11}$ and a bow-tie external cavity. ${ }^{12}$ Here, we consider a generic external ring cavity configuration such as the one shown in Fig. 1, in which the length of the active region $l_{1}$ is much shorter than the total cavity length $l_{1}+l_{2}$. We analyze both a sinusoidal and a Gaussian pulse train modulation of gain in an entire active QCL chip. It can be achieved by combining a radio frequency (RF) signal with a DC bias current, although optical modulation is also possible. ${ }^{13}$ Parasitic circuit elements can limit the high frequency response to direct modulation, yet these effects do not represent a fundamental dynamic limitation and can be mitigated using proper design and techniques developed for semiconductor lasers. 


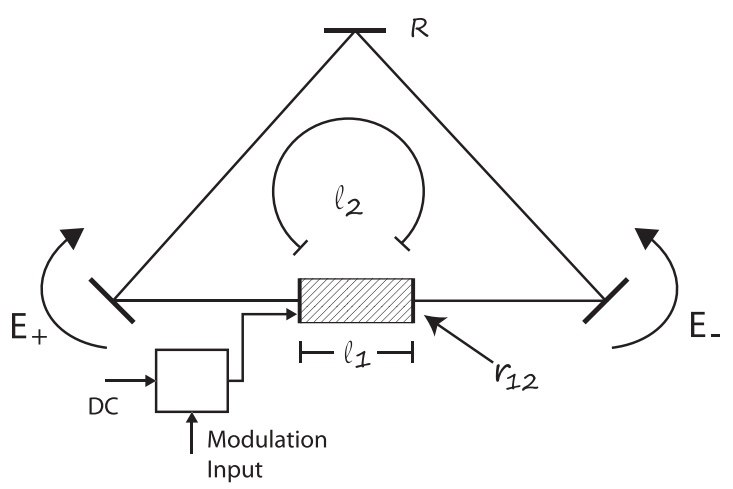

FIG. 1. A sketch of an external ring cavity laser configuration, in which a QCL chip with antireflection-coated facets of reflectivity $r_{12}$ acts as an active region.

Our analysis is based on the Maxwell-Bloch equations; details of the derivation can be found in Refs. 3 and 4. Although there are several commonly used approximations applied to simplify these equations for specific problems, e.g., the rate equation approximation, we are considering the dynamical behavior of the laser under the conditions of a strong nonlinearity, large modulation amplitude, high power, and ultrafast operation, for which we retain the full dynamics of the population and polarization. The complexity and nonlinearity of the equations require numerical methods for their solution. Although we expect unidirectional lasing in most cases, we include the population grating and both directions for the counterpropagating waves. This is necessary since it has been observed that the facet reflections, even after applying an anti-reflective coating, can induce a complex bi-directional switching behavior at critical gains and modulation frequencies, see Ref. 12.

Although more complex models of electron transport in QCLs do exist, combining them with a full spatio-temporal nonlinear dynamics of the field and polarization is beyond the current analysis and greatly complicates the model. The Maxwell-Bloch equations correctly capture the laser dynamics and reproduce the nature of the resonant nonlinearity of the gain medium, which is most important for the problem at hand.

After making a usual approximation of slowly varying amplitudes, the Maxwell-Bloch equations take the following form:

$$
\begin{aligned}
\frac{n}{c} \frac{d E_{+}}{d t} & =-\frac{d E_{+}}{d z}-i P_{+}-\alpha E_{+}, \\
\frac{n}{c} \frac{d E_{-}}{d t} & =\frac{d E_{-}}{d z}-i P_{-}-\alpha E_{-}, \\
\frac{d P_{+}}{d t} & =\frac{i}{2}\left(\Delta_{0} E_{+}+\Delta_{2}^{-} E_{-}\right)-\frac{P_{+}}{T_{2}}, \\
\frac{d P_{-}}{d t} & =\frac{i}{2}\left(\Delta_{0} E_{-}+\Delta_{2}^{+} E_{+}\right)-\frac{P_{-}}{T_{2}}, \\
\frac{d \Delta_{0}}{d t} & =\lambda(t)+i\left(E_{+}^{*} P_{+}+E_{-}^{*} P_{-}-c . c .\right)-\frac{\Delta_{0}}{T_{1}}, \\
\frac{d \Delta_{2}^{+}}{d t} & =i\left(E_{+}^{*} P_{-}-E_{-} P_{+}^{*}\right)-\left(1 / T_{1}+4 k^{2} D\right) \Delta_{2}^{+}, \\
\frac{d \Delta_{2}^{-}}{d t} & =-i\left(E_{+} P_{-}^{*}-E_{-}^{*} P_{+}\right)-\left(1 / T_{1}+4 k^{2} D\right) \Delta_{2}^{-} .
\end{aligned}
$$

The electric field is normalized by the Rabi frequency, while the other variables are normalized accordingly

$E=\frac{\tilde{E} d}{\hbar}, \quad P=\Theta \eta, \quad \Delta_{0}=\Theta \tilde{\Delta}_{0}, \quad \Delta_{2}=\Theta \tilde{\Delta}_{2}, \quad \lambda=\Theta \tilde{\lambda}$,

where the common normalization factor is $\Theta=N d^{2} k_{0} /$ $\left(\hbar \epsilon_{0} n^{2}\right)$. The variables $\tilde{E}, \eta, \tilde{\Delta}_{0}, \tilde{\Delta}_{2}$, and $\tilde{\lambda}$ correspond to the electric field envelope, amplitude of the off-diagonal element of the density matrix, population difference, population grating, and population pumping rate, respectively. Here, $N$ is the volume density of electrons, $d$ is the dipole moment, $\lambda_{0}$ is the free space wavelength, $\epsilon_{0}$ is the free space permittivity, $k_{0}=2 \pi n / \lambda_{0}$, and $n=3.3$ is the effective refractive index of the active chip. The subscripts + and - stand for the two directions of the traveling waves: clockwise and counterclockwise.

The pumping rate $\lambda(t)$ is time dependent and assumed to be directly proportional to the injection current. We will normalize it to its value at threshold for unidirectional lasing: $g(t)=\lambda / \lambda_{t h}$, where $\lambda_{t h}=2\left(\alpha-\left(1 / l_{1}\right) \ln R_{+}\right) / T_{1} T_{2}$. We will call $g(t)$ the gain or small-signal gain.

The factors $R_{+,-}$account for the total losses (transmission through the facet, beam splitting, coupling efficiency, etc.) during the round trip for the field in a given direction from one facet to another. The boundary conditions for the fields are given by

$$
\begin{aligned}
& E_{+}(t, 0)=R_{+} E_{+}\left(t-l_{2} / c, l_{1}\right)+r_{12} E_{-}(t, 0)+\Gamma(t), \\
& E_{-}\left(t, l_{1}\right)=R_{-} E_{-}\left(t-l_{2} / c, 0\right)+r_{12} E_{+}\left(t, l_{1}\right)+\Gamma(t),
\end{aligned}
$$

where the coordinate $z$ along the cavity is counted clockwise from the right-hand facet of the gain medium. Multiple reflections are neglected. The last factor $\Gamma$ is a spontaneous noise term. It is assumed to be delta-correlated and have a magnitude of $10^{-4}$ in normalized units of Eq. (2). Its magnitude is not important as long as it is small. We take $R_{-}=.48$ and $R_{+}=.5$, to account for possible asymmetries in the cavity that favor the unidirectional lasing behavior, which is consistent with the experimental results in Ref. 12. The remaining parameters and their values used during the simulations are described in Table I. The value of the diffusion coefficient in the table is taken from Ref. 4. Since in our case, the facet reflectivity is low, the diffusion term does not affect the dynamics significantly.

TABLE I. Parameters used in the simulations.

\begin{tabular}{lcc}
\hline \hline Parameter & Symbol & Value \\
\hline Active region length $^{a}$ & $l_{1}$ & $1 \mathrm{~mm}$ \\
Passive cavity length $^{a}$ & $l_{2}$ & $100 \mathrm{~cm}$ \\
Population relaxation time $_{\text {Dephasing time }}$ & $T_{1}$ & $1 \mathrm{ps}$ \\
Roundtrip time & $T_{2}$ & $0.1 \mathrm{ps}$ \\
Active region losses & $T$ & $3.35 \mathrm{~ns}(297 \mathrm{MHz})$ \\
Facet reflectivity & $\alpha$ & $10 \mathrm{~cm}^{-1}$ \\
Diffusion coefficient & $\left|r_{12}\right|^{2}$ & $1.4 \%$ \\
Wavelength & $D$ & $46 \mathrm{~cm}^{2} \mathrm{~s}^{-1}$ \\
\hline \hline
\end{tabular}


First, we analyze the situation in which the gain is modulated with a periodic train of Gaussian pulses of the following form:

$$
g\left(t+m T_{p}\right)=g_{\max } \exp \left(-\frac{(4 \log 2) t^{2}}{\tau_{p}^{2}}\right) .
$$

Here, $m$ is an arbitrary integer, which accounts for the periodicity of the signal, $T_{p}$ is the period of the pulse train, and $\tau_{p}$ is the pulse full width at half maximum (FWHM). Gaussian pulses provide more flexibility than the sinusoidal modulation, since there are two control parameters: the period and the pulse duration. Pulse formation in ring-cavity QCLs modulated with Gaussian pump pulses is illustrated in Fig. 2. Results are shown for the $E_{+}$mode that has lower loss and higher amplitude. The effect of gain modulation using short Gaussian pulses of variable duration is presented in Fig. 2(a). The gain is modulated with the periodicity equal to the

(a)

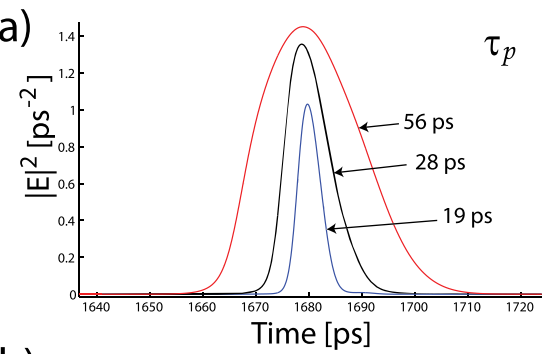

(b)

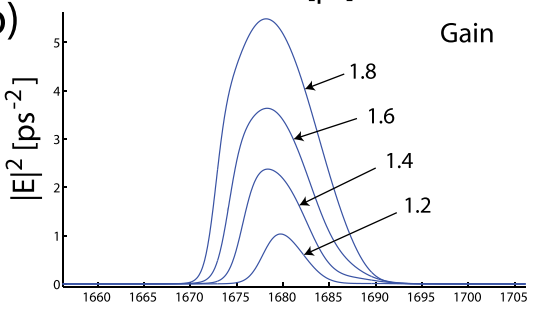

Time [ps]

\section{(c)}

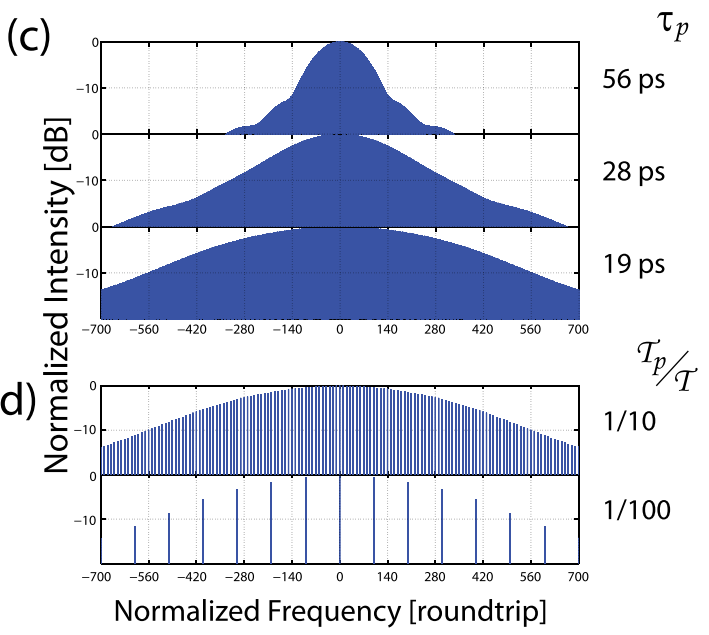

FIG. 2. (a) Output pulses, with the field expressed as the Rabi frequency according to Eq. (2), for varying pump pulse duration ( $\tau_{p}$ indicated for each pulse) and fixed repetition rate $T_{p}=T$. The output pulse duration for $\tau_{p} \simeq$ 19 ps is about 5 ps (FWHM of $|E|^{2}$ ). (b) Output pulses for varying pump pulse amplitude $g_{\max }$. Other parameters are fixed to the values $\tau_{p}=19 \mathrm{ps}$ and $T_{p}=T$. (c) Spectral response to Gaussian modulation of the gain for various pump pulse durations and with a repetition period equal to one roundtrip. (d) Spectrum of harmonic mode locking. The pump pulse duration is $\tau_{p}=19 \mathrm{ps}$, while the repetition period of the pulses $T_{p}$ is $T / 10$ (upper panel) and $T / 100$ (lower panel). roundtrip time, $T_{p}=T=l_{2} / c+n l_{1} / c$, and with a fixed value of the pump pulse amplitude: $g_{\max }=1.3$, while $\tau_{p}$ is varied. The simulations are run long enough to allow the output pulses to reach a steady state. The output pulses have the peak field amplitudes slightly higher than but comparable to the output field for a continuous wave $(\mathrm{CW})$ operation of the same laser pumped with a constant rate at the peak level $g_{C W}=g_{\max }$. An accumulation of the pulse energy beyond this limit is prevented by a fast gain relaxation. The output intensity begins to drop when the pump pulse becomes shorter than the time, it takes the light to cross the active medium, which is $n l_{1} / c \sim 10$ ps for $l_{1}=1 \mathrm{~mm}$. In this case, a circulating laser pulse experiences a lower net gain. In practice, generating the pump pulses of duration shorter than $\sim 50$ ps is a challenge.

An increase in the gain amplitude for a given pump pulse duration results in the output pulses of higher intensity and longer duration, as shown in Fig. 2(b); it does not destroy stable single-pulse operation, as opposed to active mode locking in monolithic Fabry-Perot lasers. ${ }^{2}$ We also ran simulations for longer relaxation times $T_{1,2}$ (to be published elsewhere) and observed that the pulses got asymmetric and distorted earlier (i.e., at lower field intensities) due to an increased inertia of the gain medium.

The spectrum of the laser under Gaussian modulation and under the conditions of Fig. 2(a) is presented in Fig. 2(c). There are approximately 2000 modes and the spectral width $\Delta f \sim 6 \times 10^{11} \mathrm{~Hz}$. We also calculated the modal phases using the Fast Fourier Transform of the electric field. The modes are phase-locked and have a linear phase relation. The phase relation is stable for a given current, but its slope changes depending on the gain amplitude and pump pulse duration, which can be also inferred from the change of the shape of the pulses in time domain. For long pump pulses, it becomes piecewise linear. The detailed study will be published elsewhere.

If the gain pulse half-width, $\tau_{p}$ is maintained fixed, while changing the modulation period $T_{p}$, the resulting pulses have a higher repetition rate. The total spectral width remains the same, but only one of every $M$ modes is excited, where the value of integer $M=T / T_{p}$ can be controlled. The harmonic spectrum under such modulation is presented in Fig. 2(d). Note that $M$ has to be integer, otherwise the laser intensity drops to the level of noise.

Next consider the response to a sinusoidal gain modulation with a DC offset component

$$
g(t)=g_{0}+g_{s} \sin \left(2 \pi t / T_{p}\right)
$$

Small-amplitude modulation with $g_{0}=1.3$ and $g_{s}=0.1$ shows a $3 \mathrm{~dB}$ cutoff frequency of $f_{c} \approx 20 \mathrm{GHz}$, which is consistent with the results from Refs. 14 and 15 and can be verified analytically. For a deep sinusoidal modulation, we use $g_{0}=0.8$ and $g_{s}=0.65$, while changing the modulation pe$\operatorname{riod} T_{p}$. The laser demonstrates pulsed operation and the pulse duration monotonically decreases with decreasing $T_{p}$. In order to obtain pulse duration in a few ps range, $T_{p}$ has to be much shorter than the round-trip time $T$, as shown in Fig. 3. This results in harmonic mode locking with the spectra similar to those shown in Fig. 2(d). However, reaching the 


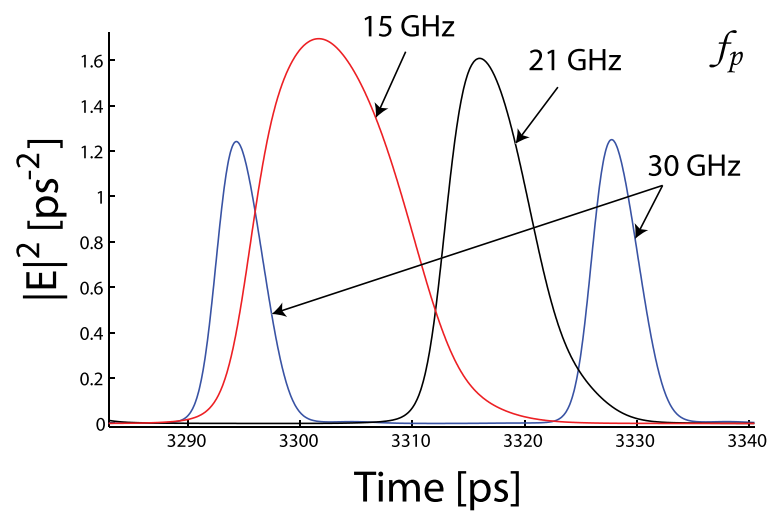

FIG. 3. Output pulses in a sinusoidally modulated ERC-QCL for different modulation periods $T_{p}$ and corresponding frequencies $1 / T_{p}$. The field is expressed as the Rabi frequency according to Eq. (2). The output pulse duration is about 4 ps for a $30 \mathrm{GHz}$ modulation.

modulation frequency above $25 \mathrm{GHz}$ is a challenge. Besides, sinusoidal modulation requires a higher pumping power than the modulation with Gaussian pulses.

In conclusion, we show that gain modulation of external ring cavity QCLs at the round-trip frequency or its multiples should result in a robust active mode locking of a large number of longitudinal cavity modes, picosecond pulse generation, and phase-locked frequency combs in the mid-infrared. The maximum pulse peak power is reached when the pulse experiences amplification along its entire transit in the gain medium. For this to happen, the time during which the gain is above threshold $\left(\sim \tau_{p}\right.$ in our simulation) has to be longer than the pulse transit time in the gain medium $\tau_{t}=n l_{1} / c$. For $\tau_{p}<\tau_{t}$, shorter optical pulses are obtained at the expense of the peak power. To obtain shorter output pulses, while still maintaining a maximum peak power, one needs to decrease both $\tau_{t}$ and $\tau_{p}$, while keeping $\tau_{p} \sim \tau_{t}$. In practice, their duration will be limited by the technical difficulty of synthesizing a very short current pulse. The proposed scheme can be modified by adding a nonlinear loss element in the external cavity to help shaping the optical pulse. In this way, a less technically demanding RF pulse synthesis would be required.

This work has been supported in part by NSF Grant Nos. ECCS 1230517 and EEC-0540832. T.S.M. was supported by the NSF Graduate Student Fellowship.

${ }^{1}$ R. Paiella, F. Capasso, C. Gmachl, C. G. Bethea, D. L. Sivco, J. N. Baillargeon, A. L. Hutchinson, and A. Y. Cho, Appl. Phys. Lett. 75, 2536 (1999).

${ }^{2}$ C. Y. Wang, L. Kuznetsova, V. M. Gkortsas, L. Diehl, F. X. Kärtner, M. A. Belkin, A. Belyanin, X. Li, D. Ham, H. Schneider et al., Opt. Express 17, 12929 (2009).

${ }^{3}$ A. Gordon, C. Y. Wang, L. Diehl, F. X. Kärtner, A. Belyanin, D. Bour, S. Corzine, G. Höfler, H. C. Liu, H. Schneider et al., Phys. Rev. A 77, 053804 (2008).

${ }^{4}$ V.-M. Gkortsas, C. Wang, L. Kuznetsova, L. Diehl, A. Gordon, C. Jirauschek, M. A. Belkin, A. Belyanin, F. Capasso, and F. X. Kärtner, Opt. Express 18, 13616 (2010).

${ }^{5}$ S. Barbieri, M. Ravaro, P. Gellie, G. Santarelli, C. Manquest, C. Sirtori, S. P. Knahna, E. H. Linfield, and A. G. Davies, Nat. Photonics 5, 306 (2011).

${ }^{6}$ J. Freeman, J. Maysonnave, N. Jukam, P. Cavali, K. Maussang, H. Beere, D. Ritchie, J. Mangeney, S. Dhillon, and J. Tignon, Appl. Phys. Lett. 101, 181115 (2012).

${ }^{7}$ A. Hugi, G. Villares, S. Blaser, H. C. Liu, and J. Faist, Nature 492, 229 (2012).

${ }^{8}$ N. Yu, L. Diehl, E. Cubukcu, D. Bour, S. Corzine, G. Höfler, A. K. Wójcik, K. B. Crozier, A. Belyanin, and F. Capasso, Phys. Rev. Lett. 102, 013901 (2009).

${ }^{9}$ A. K. Wójcik, N. Yu, F. Capasso, and A. Belyanin, J. Mod. Opt. 58, 727 (2011).

${ }^{10}$ A. K. Wójcik, N. Yu, L. Diehl, F. Capasso, and A. Belyanin, Phys. Rev. Lett. 106, 133902 (2011).

${ }^{11}$ C. C. Nshii, C. N. Ironside, M. Sorel, T. J. Slight, S. Y. Zhang, D. G. Revin, and J. W. Cockburn, Appl. Phys. Lett. 97, 231107 (2010).

${ }^{12}$ P. Malara, R. Blanchard, T. Mansuripur, A. Wójcik, A. Belyanin, T. Edamura, S. Furuta, K. Fujita, M. Yamanishi, P. de Natale et al., Appl. Phys. Lett. 102, 141105 (2013).

${ }^{13}$ G. Chen, R. Martini, S. Wook Park, C. G. Bethea, I.-C. A. Chen, P. D. Grant, R. Dudek, and H. C. Liu, Appl. Phys. Lett. 97, 011102 (2010).

${ }^{14}$ B. Meng and Q. J. Wang, Opt. Express 20, 1450 (2012).

${ }^{15}$ M. Haldar, IEEE J. Quantum Electron. 41, 1349 (2005). 